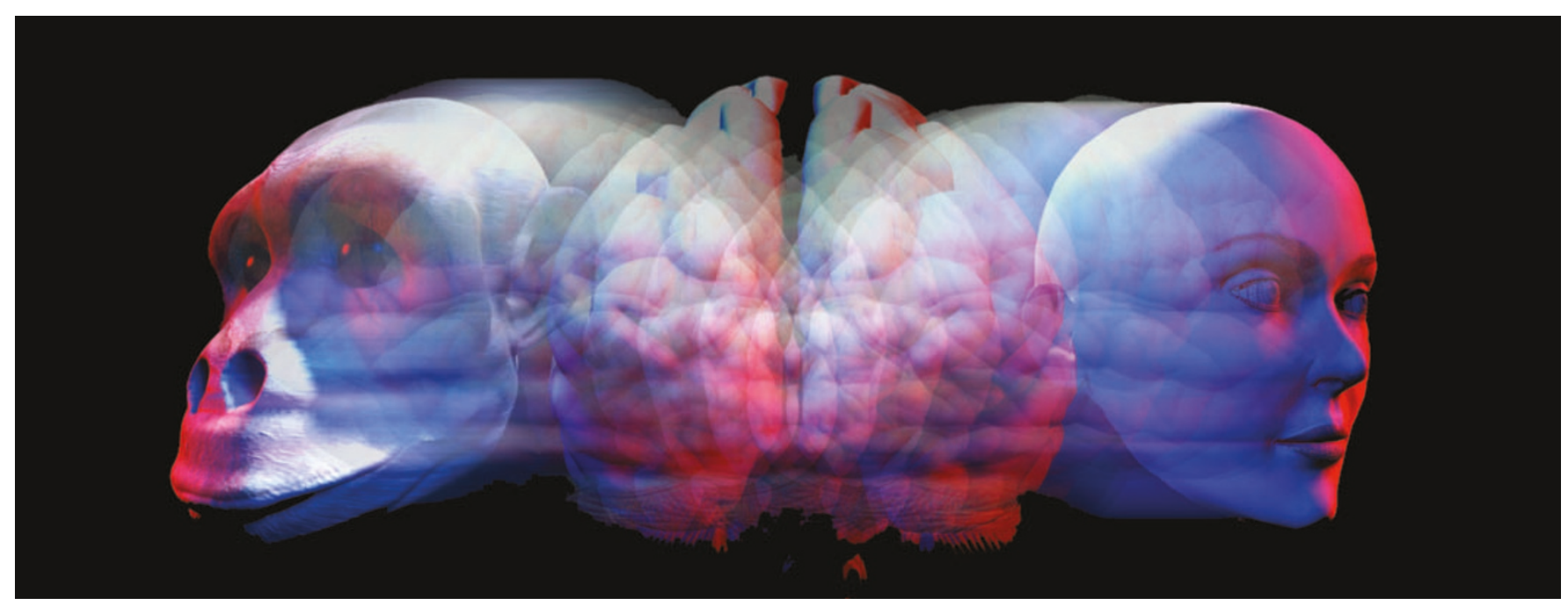

\title{
The human factor
}

\section{What is it that makes us different from other animals?}

\section{So You Think You're Human?}

A Brief History of Humankind

by Felipe Fernández-Armesto

Oxford University Press: 2004. 208 pp.

$£ 14.99, \$ 23$

\section{The Human Story: A New History}

\section{of Mankind's Evolution}

by Robin Dunbar

Faber \& Faber: 2004. 224 pp. 112.99

\section{David L. Hull}

Writing for a popular audience is not quite as easy as authors generally think it is. Most popularizations are much too technical and not sufficiently engrossing. Both Felipe Fernández-Armesto and Robin Dunbar, on the other hand, have gauged their audience just right. They have simplified without falsifying and have picked a topic that people find endlessly fascinating - the nature of human nature.

These two books also complement each other nicely. Dunbar begins with the fossil record and then leaps ahead to the present. Most of his discussion concerns current ideas about human beings as we have studied them scientifically. Fernández-Armesto devotes most of his book to filling in the gap between our fossil ancestors and the present, expanding his investigations to include both the history and the prehistory of humans at a wide variety of places and times. Those of us living right now in the West assume there is such a thing as human nature that makes us different from other animals, and that this belief stretches back indefinitely in time. We might disagree about what this nature is, but we take for granted that it exists. As it turns out, a belief in human nature is of recent vintage and is not all that widespread.

In So You Think You're Human?FernándezArmesto shows exactly how mistaken this chauvinistic belief actually is. People in the West think that species can be distinguished from each other quite easily. But in the past, people in various cultures have thought that the boundaries between species are both permeable and fuzzy. In some cultures a wide variety of entities have been treated as if they should be classified with us. Conversely, some of those we regard as unproblematically human were in other societies not considered human at all. For example, when the neighbours of pygmies killed and ate them, they did not think they were doing anything different from killing and eating wild boar or monkeys. If they killed and ate each other, that would be cannibalism; doing the same to pygmies was not.

Although both Fernández-Armesto and Dunbar are interested in the same issue human nature - they approach it differently. Fernández-Armesto overwhelms the reader with example after example, whereas Dunbar presents more careful, coherent arguments. Both authors discuss many of the same things: bodily differences such as the plantigrade (flat to the floor) foot, opposable thumbs, bipedal gait and big brains; mental differences in intelligence, speech, imagination, tool manufacture and use, fire and cooking; and finally social differences in culture, religion, music, art, reason, use of medicine, social learning and the formation of social groups.

Fernández-Armesto concludes that the differences between us and other primates are not differences of kind but of degree, albeit an enormous degree. Although Dunbar is sympathetic, he is forced to the opposite conclusion. The ultimate criterion for Dunbar is intentionality and an accompanying theory of mind. Until the age of four or so, human children have only a single level of intentionality. They are aware of their own intentions, but they cannot distinguish between their own intentions and those of others. But after this crucial period, they can. As they mature, the levels of intentionality increase until they can understand that when Shakespeare wrote Twelfth Night, "he intended [1] that his audience should realise [2] that the much derided Malvolia believed [3] that his mistress Olivia wanted [4] to marry him instead of his being her servant."

Chimpanzees and autistic children are not able to develop a theory of mind. Chimpanzees are "on the brink", says Dunbar, but that is all. This dispute does not seem to afford any sort of resolution. One side sees only differences in degree. The other side sees a difference in kind, and if not in kind, at least, massive differences in degree.

Fernández-Armesto ends his book by concluding that we need to rethink how we conceive of the living world, in particular the human species. I could not agree more. In the recent past, at least, everyone who has addressed this question has assumed that species are kinds defined in terms of characteristics - the task was to find the right ones. But what if we do not treat species in this way at all but as individuals? Before the acceptance of evolutionary theory, species seemed to be paradigmatic kinds, but with the introduction of evolutionary theory, new dimensions were introduced: place and time. For selection to work, descent is required, and that is a spatiotemporal relation.

If we accept the role of variation and selection in the evolution of species, then descent must take priority over similarity. The problem is that we glide too readily over fundamentally different notions. For example, species have boundaries, but two quite different sorts of boundary are being 
invoked, only one of which is spatiotemporal. Biogeographers trace the spatiotemporal boundaries of species, discovering for example that catalpa trees are indigenous to the Wabash Valley. The other sorts of boundaries are conceptual and concern definitions. All triangles, and only triangles, have three sides.

Although not all species reproduce sexually, those that do so produce a genealogical nexus, and species are chunks of it. At best, the way that characteristics are distributed is secondary to descent. In referring to species as the groupings that evolve by variation and selection, I do not mean to imply that this is the only way of defining species. Lots of people do it in lots of different ways, but Fernández-Armesto and Dunbar both introduce Darwin and evolution. So they need to be made aware of one quite prevalent way of construing species, including humans, especially as it provides rather a different perspective on the human species. FernándezArmesto devotes his final chapter to issues surrounding morality. Once again, as he sees it, the issue is characteristics. Which characteristics are relevant to morality, and which organisms exhibit them? What should we do with non-human organisms that have the characteristics that we use to confer moral rights?

From the evolutionary perspective, how- ever, the issue is genealogy, not distributions of characteristics. Even though some pigs may seem brighter than some people, pigs do not belong to the same chunk of the genealogical nexus as people. Evolutionary theory is inherently species-ist. If we discovered that dolphins have sufficiently welldeveloped language skills that we could strike up conversations with them, they would still be dolphins and we would still be humans. Social and moral problems would arise, but as biological species we would remain unique and distinct.

David L. Hull is emeritus professor at the Department of Philosophy, Northwestern University, Evanston, Illinois 60208-1315, USA.

\section{Walking on their ribs}

\section{Geoffroy Saint-Hilaire: A Visionary Naturalist \\ by Hervé Le Guyader, \\ transl. Marjorie Grene \\ University of Chicago Press: 2004. 302 pp.

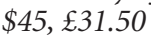

\section{Nick Hopwood}

In evolutionary terms, the most remarkable discoveries of developmental biology in the 1980s and 1990s were that the molecular mechanisms of anteroposterior axis formation are shared across most of the animal kingdom, and that vertebrates form a dorsoventral axis in a similar way to insects, only upside-down. This axial inversion had a special appeal because it seemed to confirm an old and much-derided view. Its first proponent was the French zoologist Étienne Geoffroy Saint-Hilaire, subject of this book by Hervé Le Guyader.

In 1830, Geoffroy faced his one-time friend and long-term colleague Georges Cuvier at the Muséum d'Histoire Naturelle in Paris, in one of the most famous controversies in the history of science. Cuvier, the most powerful comparative anatomist of the age, had divided the animal kingdom into four completely separate branches: vertebrates, articulates (largely arthropods and annelids), molluscs and radiates (echinoderms, cnidarians and various other groups). Even within these divisions, he allowed structural similarity to result solely from the same functional demands.

Geoffroy, by contrast, taught that function did not really matter, nor even form; what counted were the connections between the parts. He founded a 'philosophical' anatomy on 'analogy' (homology, to us), and pushed the idea that all animals are built to a single plan. Having established a common scheme for vertebrates, in 1820 he extended it to the articulates. Insects, he pronounced,

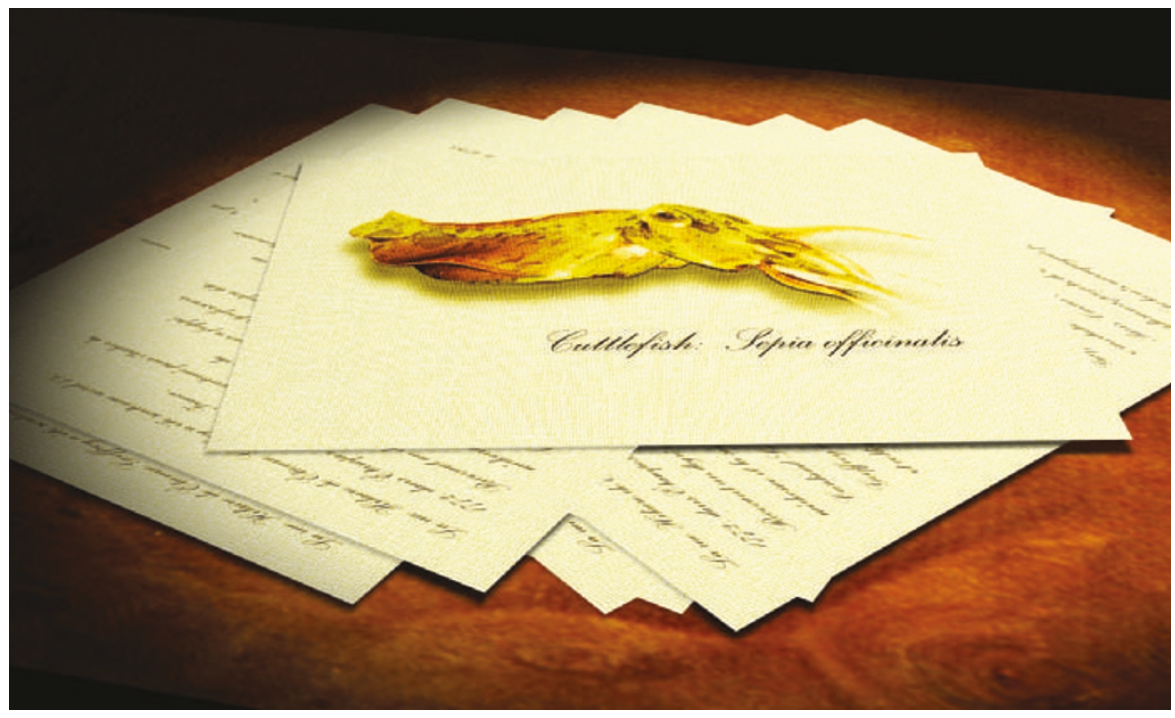

are vertebrates that live within their vertebral columns and walk on their ribs.

Cuvier restrained himself until a decade later, when Geoffroy exploited two young naturalists' suggestion that cuttlefish, representing molluscs, were like vertebrates doubled back on themselves. The simmering dispute now boiled over into a confrontation on the floor of the Academy of Sciences in Paris that captivated the learned world and newspaper-reading public alike.

As Toby Appel showed in The CuvierGeoffroy Debate (Oxford University Press, 1987), much more than cephalopod anatomy was at stake. Cuvier's functionalism opposed Geoffroy's morphology. The austere, logical Protestant fought the intuitive, impetuous, romantic Deist. Cuvier's factdriven, establishment science was pitted against Geoffroy's broad speculation and alliances with progressive forces beyond the academy. Cuvier is usually said to have won, and on the narrow issue he did, but Geoffroy's philosophical anatomy was more influential than used to be thought. For leading naturalists in the next generations, both positions seemed too extreme. In finding a resolution, homology was made into evidence of darwinian evolution - and some darwinists argued that the vertebrates originated from annelids by inversion of the dorsoventral axis.

Le Guyader's book, first published in French six years ago, offers a judicious selection of Geoffroy's works, each with a short introduction. These texts have never been translated before because nineteenth-century British naturalists read them in French, and because by the middle of the century Geoffroy had been marginalized. We have the preliminary discourses to both volumes of his Anatomical Philosophy; the first of three treatises on the organization of insects, which deals with the anteroposterior axes of insects and vertebrates; the recently muchcited review, "General considerations on the vertebra", with its reflections on dorsoventral organization and the figure of the insideout and upside-down lobster; and the whole of the Principles of Zoological Philosophy, Geoffroy's record of the 1830 debate.

Anglophone biologists and historians of science will be glad to have these scarce and important works so readily available. Some additional editorial work might, though, have produced more scholarly and userfriendly texts. Martin Rudwick's translations in Georges Cuvier, Fossil Bones and Geological 